\title{
Ground-state fidelity at first-order quantum transitions
}

\author{
Davide Rossini and Ettore Vicari \\ Dipartimento di Fisica dell'Università di Pisa and INFN, Largo Pontecorvo 3, I-56127 Pisa, Italy
}

(Received 9 July 2018; revised manuscript received 24 September 2018; published 26 December 2018)

\begin{abstract}
We analyze the scaling behavior of the fidelity, and the corresponding susceptibility, emerging in finite-size many-body systems whenever a given control parameter $\lambda$ is varied across a quantum phase transition. For this purpose we consider a finite-size scaling (FSS) framework. Our working hypothesis is based on a scaling assumption of the fidelity in terms of the FSS variables associated with $\lambda$ and its variation $\delta \lambda$. This framework entails the FSS predictions for continuous transitions and enables one to extend them to first-order transitions, where the FSS becomes qualitatively different. The latter is supported by analytical and numerical analyses of the quantum Ising chain along its first-order quantum transition line, driven by an external longitudinal field.
\end{abstract}

DOI: 10.1103/PhysRevE.98.062137

\section{INTRODUCTION}

Quantum transitions (QTs) in many-body systems are related to significant changes of the ground state and lowexcitation properties, induced by small variations of a driving parameter $[1,2]$. They are continuous when the ground state of the system changes continuously at the transition point, and correlation functions develop a divergent length scale. Instead, they are of first order when the ground-state properties are discontinuous across the transition point, generally arising from level crossings in the infinite-volume limit. In view of their key role played in several contexts of modern statistical mechanics, quantum information, and condensed matter physics, it is of crucial importance to devise suitable tools for a proper characterization of their main features. To this purpose, different quantum-information-based concepts have been recently put forward, in order to spotlight ground-state variations at QTs, such as the entanglement, as well as the fidelity and its susceptibility [3-5]. The net advantage of these approaches is that they do not rely on the identification of an order parameter with the corresponding symmetry-breaking pattern.

In particular, the fidelity quantifies the overlap between the ground states of quantum systems sharing the same Hamiltonian but associated with different Hamiltonian parameters [3-5]. The concept of the fidelity and, more generally, of the geometric tensor has recently gained considerable attraction in the field of quantum information and computation. The reason is related to its fundamental importance as a basic tool to analyze the variations of a given quantum state in the Hilbert space. The usefulness of the fidelity as a tool to distinguish quantum states can be traced back to Anderson's orthogonality catastrophe [6]: The overlap of two many-body ground states corresponding to Hamiltonians differing by a small perturbation vanishes in the thermodynamic limit. It is thus tempting to quantify how this paradigm gets realized in many-body systems at QTs, where significantly different behaviors are expected with respect to systems in normal conditions. Besides that, the fidelity susceptibility covers a central role in quantum estimation theory $[7,8]$, being proportional to the Fisher information. The latter indeed quantifies the inverse of the smallest variance in the estimation of the varying parameter such that, in proximity to QTs, metrological performances are believed to drastically improve $[9,10]$.

The past decade has seen the birth of intense theoretical activity focusing on the behavior of the fidelity and of the corresponding susceptibility (more generally, of the geometric tensor) [11-13] at continuous QTs (CQTs). In quantum manybody systems, the establishment of a nonanalytic behavior has been exploited to evidence CQTs in several different contexts, which have been deeply scrutinized both analytically and numerically. We quote, for example, free-fermion models [14-18], interacting spin [19-25] and particle models [26-32], and systems presenting peculiar topological [33-35] and nonequilibrium steady-state transitions [36,37]. However, a characterization of first-order QTs (FOQTs) in this context is still missing, despite the fact that they are of great phenomenological interest. Indeed, they occur in a large variety of many-body systems, including quantum Hall samples [38], itinerant ferromagnets [39], heavy fermion metals [40-42], disordered systems [43,44], and infinite-range models [45,46].

We also stress that, to achieve a deep understanding of QTs from the outcomes of numerical simulations or of quantum-simulation experiments, it is fundamental to exploit the impact of having a finite size. The natural theoretical context where to set up the analysis is the finite-size scaling (FSS) framework, which has been proven to be effective in proximity to any type of QT. Indeed, the emergence of FSS limits has been predicted both for CQTs $[1,2,47]$ and for FOQTs [48], as well as to describe the quantum dynamics of finite-size many-body systems subject to time-dependent perturbations $[49,50]$. This formalism has been successfully applied in a variety of systems, for observables such as the free energy, the energy gaps of the first low-lying levels, and correlation functions, as well as in the presence of different boundary conditions [51-53]. Recently, it has been also used to study quantum-information-based concepts, such as entanglement $[3,47,54]$ and other indicators of quantum correlations [55]. Some results for the FSS of the fidelity have been obtained in specific situations at CQTs, such as for the quantum Ising chain in a transverse field (see the 
discussion in Sec. III A), and by means of quite complicated methods [4].

In this paper we present a unified picture for the scaling behavior of the fidelity and its susceptibility, emerging in many-body systems whenever a given control parameter is varied across any type of QT. Since ground-state overlaps related to variations of the Hamiltonian parameters are naturally defined only for finite quantum systems whose groundstate wave functions are normalizable, we consider finite-size systems and focus on the asymptotic large-volume behavior of the fidelity, defined in the limit of small variations of the parameter driving the QT. The FSS theory constitutes the optimal framework to discuss this issue. It turns out to be especially effective to provide the power or exponential laws describing the size dependence of fidelity and its susceptibility when the system is driven across a QT. In particular we discuss FOQTs.

Assuming that the fidelity of finite systems is an analytic function of the relevant scaling variables associated with the driving parameter and its variation, we put forward a FSS behavior that entails the expected power-law divergences associated with CQTs while enabling us to extend the analysis to FOQTs. In the latter, the type of divergence is controlled by the closure of the gap between the two lowest energy levels, being exponential in most of the cases. A scaling theory for the fidelity provides a simple and intuitive route towards a complete understanding of the behaviors of finite-size manybody systems at CQTs and FOQTs, which is mandatory to distinguish them and obtain correct interpretations of experimental and numerical results at QTs.

The paper is organized as follows. In Sec. II we discuss the theory underlying our FSS framework for the fidelity and its susceptibility, holding whenever a many-body system undergoes a QT. Our predictions are then verified in Sec. III for the paradigmatic quantum Ising model driven by an additional external longitudinal field, exhibiting a rich phase diagram. In this context, we focus on both CQTs (Sec. III A) and FOQTs (Sec. III B) with different boundary conditions. A summary of our results, together with the perspectives, is given in Sec. IV.

\section{FINITE-SIZE SCALING OF THE FIDELITY AND ITS SUSCEPTIBILITY}

\section{A. Fidelity and its susceptibility}

We define our setting by considering a $d$-dimensional quantum many-body system of size $L^{d}$, with Hamiltonian

$$
H(\lambda)=H_{0}+\lambda H_{I},
$$

where $\left[H_{0}, H_{I}\right] \neq 0$ and the parameter $\lambda$ drives the QT located at $\lambda=0$. The fidelity

$$
F(\lambda, \delta \lambda, L) \equiv\left|\left\langle\Psi_{0}(\lambda+\delta \lambda, L) \mid \Psi_{0}(\lambda, L)\right\rangle\right|
$$

is a geometrical object that can be used to monitor the changes of the ground-state wave function $\left|\Psi_{0}(\lambda, L)\right\rangle$ when varying the control parameter $\lambda$ by a small amount $\delta \lambda$ around its transition value. Assuming $\delta \lambda$ sufficiently small, one can expand Eq. (2) in powers of $\delta \lambda$ [4],

$$
F(\lambda, \delta \lambda, L)=1-\frac{1}{2}\left(\delta \lambda^{2}\right) \chi_{F}(\lambda, L)+O\left(\delta \lambda^{3}\right),
$$

where $\chi_{F}$ defines the fidelity susceptibility. The cancellation of the linear term of the expansion is essentially related to the fact that the fidelity is bounded, i.e., $F \leqslant 1$. Standard perturbation theory allows us to also write $\chi_{F}$ as [4]

$$
\chi_{F}(\lambda, L)=\sum_{n>0} \frac{\left|\left\langle\Psi_{n}(\lambda, L)\left|H_{I}\right| \Psi_{0}(\lambda, L)\right\rangle\right|^{2}}{\left[E_{n}(\lambda, L)-E_{0}(\lambda, L)\right]^{2}},
$$

where $\left|\Psi_{n}(\lambda, L)\right\rangle$ is the Hamiltonian eigenstate corresponding to the eigenvalue $E_{n}(\lambda, L)$ (notice that the index $n=0$ labels ground-state quantities). As we will see below, the interplay between $\lambda$ and $L$ at QTs can be suitably described within FSS frameworks at both CQTs [2,47] and FOQTs [48].

\section{B. Finite-size scaling at continuous quantum transitions}

Singular behaviors at QTs are observed in the infinitevolume limit. If the size $L$ of the system is finite, all properties are generally analytic as a function of the quantity driving the transition. However, around the transition point, low-energy thermodynamic quantities and large-scale structural properties undergo peculiar FSS behaviors depending only on the nature and on the general properties of the transition. Understanding these finite-size properties is of primary importance for a correct and unambiguous interpretation of experimental or numerical data when phase transitions are investigated in relatively small systems (see, e.g., Refs. [56-59]) or in particle systems trapped by external forces, as in cold-atom experiments (see, e.g., Ref. [60]).

The modern theory of FSS delineates the standard road map to investigate these issues at phase transitions. It was originally developed in the context of critical phenomena and formulated in the classical framework [56,61]. At continuous transitions, FSS is observed when the length scale $\xi$ of the critical modes becomes comparable to $L$. For large values of $L$, this regime presents universal features, shared by all systems whose transition belongs to the same universality class. Analogous behaviors emerge at CQTs $[2,47]$, where the FSS framework allows one to characterize the finite-size dependence of the low-energy properties of quantum manybody systems, in particular the low-excitation spectrum, the correlation functions, etc. The critical behavior is generally characterized by power laws, with universal exponents determined by the universality class of the CQT. They do not depend on the microscopic details of the quantum model, but only on some global properties, such as the spatial dimension, the symmetry, and the nature of the interactions (whether they are short range or long range). In particular, relevant universal exponents are the renormalization-group (RG) dimension $y_{\lambda}$ of the parameter $\lambda$ driving the transition and the dynamic exponent $z$ associated with the scaling behavior of the gap, i.e., the energy difference of the lowest states [1].

The FSS limit is generally obtained at large $L$, keeping an appropriate combination $\kappa$ of $\lambda$ and $L$ fixed. At CQTs, this is generally given by [47]

$$
\kappa=\lambda L^{y_{\lambda}} .
$$

Generic observables $\mathcal{O}$ behave as $[2,47]$

$$
\mathcal{O}(\lambda, L) \approx L^{-y_{o}} f_{\mathcal{O}}(\kappa)
$$


where $y_{o}$ is the RG dimension associated with $\mathcal{O}$ and $f_{\mathcal{O}}(\kappa)$ a scaling function. Note that the universal power laws at CQTs do not depend on the boundary conditions, which only affect the scaling functions.

The temperature $T$ gives rise to an additional relevant perturbation at CQTs. Within the FSS framework, it is taken into account by adding a further dependence of the scaling functions on the scaling variable $[1,2]$

$$
\tau \sim T / \Delta_{0}(L), \quad \Delta_{0}(L) \sim L^{-z},
$$

where $\Delta_{0}(L)$ is the energy difference of the lowest states at the transition point of CQTs and $z$ is the dynamic exponent.

We are now in the position to discuss the scaling behavior of the fidelity $F(\lambda, \delta \lambda, L)$ and its susceptibility $\chi_{F}(\lambda, L)$, assuming that both $\lambda$ and $\lambda+\delta \lambda$ are sufficiently small to be in the transition region. We conjecture that the zero-temperature scaling is given by

$$
F(\lambda, \delta \lambda, L) \approx \mathcal{F}(\kappa, \delta \kappa),
$$

where $\delta \kappa$ is the variation of $\kappa$ corresponding to $\delta \lambda$. The scaling relation (8) is quite natural, noting that $F(\lambda, 0, L)=1$ and that a regular expansion around $\delta \lambda=0$ is expected at finite volume. Correspondingly, we expect $\mathcal{F}(\kappa, 0)=1$ and a regular behavior around $\delta \kappa=0$. The FSS of $\chi_{F}$ can be immediately derived from Eq. (8), by expanding $\mathcal{F}$ in powers of $\delta \kappa$,

$$
\mathcal{F}(\kappa, \delta \kappa)=1-\frac{1}{2}\left(\delta \kappa^{2}\right) \mathcal{F}_{2}(\kappa)+O\left(\delta \kappa^{3}\right),
$$

and matching it with Eq. (3),

$$
\chi_{F}(\lambda, L) \approx(\delta \kappa / \delta \lambda)^{2} \mathcal{F}_{2}(\kappa) .
$$

This implies

$$
\chi_{F}(\lambda, L) \approx L^{2 y_{\lambda}} \mathcal{F}_{2}(\kappa)
$$

We stress that this obtained FSS power law perfectly agrees with earlier (apparently more involved) derivations, which have been obtained by means of alternative scaling arguments [4]. ${ }^{1}$ However, an important feature of our derivation is that the validity of Eq. (10) can be extended to FOQTs as well, by inserting the appropriate scaling variable $\kappa$ (discussed below). In such a case, for transitions based on the avoided crossing of two levels, the conjecture (8) can be straightforwardly justified by means of a simple calculation on the effective Hamiltonian as well (see the Appendix).

\section{Finite-size scaling at first-order quantum transitions}

Finite-size scaling behaviors also develop at FOQTs, although with significant differences [48]. In particular, they turn out to be more sensitive to the boundary conditions, which may give rise to different functional dependences of the corresponding scaling variable $\kappa$, leading to both exponential and power laws.

First-order quantum transitions generally arise from level crossings. However, level crossings can only occur in the

\footnotetext{
${ }^{1}$ To compare with Refs. [13,20], simply use the scaling relation [1] $y_{\lambda}=d+z-y_{H_{I}}$, where $z$ is the dynamic exponent and $y_{H_{I}}$ is the RG dimension of $H_{I} / L^{d}$.
}

infinite-volume limit (in the absence of particular conservation laws). In a finite system, the presence of a nonvanishing matrix element among these states lifts the degeneracy, giving rise to the phenomenon of avoided level crossing. Here the FSS is controlled by the energy difference $\Delta(\lambda, L)$ of the avoiding levels, in particular by

$$
\Delta_{0}(L) \equiv \Delta(\lambda=0, L) .
$$

The appropriate FSS variable is generally given by [48]

$$
\kappa \sim \frac{E_{\lambda}(\lambda, L)}{\Delta_{0}(L)},
$$

$E_{\lambda}$ being the energy variation associated with the $\lambda$ term (we assume $E_{\lambda}=0$ at the transition point). The FSS limit is defined by the large- $L$ limit, keeping $\kappa$ fixed. However, it is important to remark that the FSS at FOQTs is more complex than that at CQTs, because it may significantly depend on the boundary conditions [48,51-53]: The gap $\Delta_{0}(L)$ may depend on the size $L$ either exponentially (as it occurs in typical situations) or even as a power law. As a matter of fact, the FOQT scenario based on the avoided crossing of two levels is not always realized, depending on the boundary conditions (discussed below); indeed, in some cases the energy difference $\Delta_{0}(L)$ of the lowest levels may even show a power-law dependence on $L$. However, as we will see, the scaling variables $\kappa$ obtained using the corresponding $\Delta_{0}(L)$ turn out to be appropriate as well.

In order to derive the scaling behavior of the fidelity and its susceptibility [cf. Eqs. (2) and (3)] we can repeat the scaling arguments of Sec. II B done at CQTs. Therefore, assuming again that both $\lambda$ and $\lambda+\delta \lambda$ are sufficiently small to be in the transition region, we obtain Eqs. (8) and (10) as well, but with the appropriate scaling variable $\kappa$ given now by Eq. (13). In particular, for FOQTs we obtain

$$
\chi_{F}(\lambda, L) \approx \Delta_{0}(L)^{-2}\left(\partial E_{\lambda} / \partial \lambda\right)^{2} \mathcal{F}_{2}(\kappa) .
$$

We note that at FOQTs the finite-size dependence of the fidelity susceptibility appears to be closely connected with the size dependence of the energy difference of the lowest levels. Since the gap can be exponentially suppressed for some types of boundary conditions, such as periodic or equal and fixed boundary conditions, for which $\Delta_{0}(L) \sim e^{-a L^{d}}$, in such cases we expect corresponding exponentially large behaviors for the fidelity susceptibility $\chi_{F} \sim e^{c L^{d}}$ at the transition point (Secs. III B 1 and III B 3). For other types of boundary conditions, such as antiperiodic boundary conditions, for which $\Delta_{0}(L) \sim L^{-b}$, we expect a power-law behavior of the fidelity susceptibility with $L$ (Sec. III B 2 ), as it happens in proximity to CQTs.

\section{Finite-size scaling at finite temperature}

The above FSS framework, both for CQTs and for FOQTs, can be generalized to a finite temperature $T$ as well [4]. In such a case, the quantum system is described by the density matrix

$$
\rho_{\lambda} \equiv \rho(\lambda, T, L)=Z^{-1} \sum_{n} e^{-E_{n} / k_{B} T}\left|\Psi_{n}\right\rangle\left\langle\Psi_{n}\right|,
$$


where $Z=\sum_{n}\left\langle\Psi_{n}\left|e^{-E_{n} / k_{B} T}\right| \Psi_{n}\right\rangle$ defines the partition function. The fidelity between two mixed states can be defined as [62]

$$
F(\lambda, \delta \lambda, T, L)=\operatorname{Tr} \sqrt{\sqrt{\rho_{\lambda}} \rho_{\lambda+\delta \lambda} \sqrt{\rho_{\lambda}}}
$$

which reduces to Eq. (2) for $T \rightarrow 0$. The corresponding fidelity susceptibility can be extracted analogously to Eq. (3). At a QT, the $T=0$ scaling (8) can be straightforwardly extended to keep account of the temperature, by adding a further scaling variable $\tau=T / \Delta_{0}(L)$, so that

$$
F(\lambda, \delta \lambda, T, L) \approx \mathcal{F}(\kappa, \delta \kappa, \tau) .
$$

This scaling equation holds at both CQTs and FOQTs, with the appropriate definitions of scaling variables. In particular, $\tau=T / \Delta_{0}(L) \sim T L^{z}$ at CQTs, where $z$ is the dynamic exponent.

\section{RESULTS FOR THE QUANTUM ISING CHAIN}

We now verify the above general FSS predictions by presenting analytical and numerical evidence for the paradigmatic one-dimensional quantum Ising model in the presence of transverse and longitudinal fields. Its Hamiltonian reads

$$
H_{\mathrm{Is}}=-J \sum_{\langle i, j\rangle} \sigma_{i}^{(3)} \sigma_{j}^{(3)}-g \sum_{i} \sigma_{i}^{(1)}-h \sum_{i} \sigma_{i}^{(3)},
$$

where $\sigma^{(k)}$ are the Pauli matrices, the first sum is over all bonds connecting nearest-neighbor sites $\langle i, j\rangle$, and the other sums are over the $L$ sites. Hereafter we assume $\hbar=k_{B}=1$, $J=1$, and $g>0$.

At $g=1$ and $h=0$, the model undergoes a CQT belonging to the two-dimensional Ising universality class, separating a disordered phase $(g>1)$ from an ordered $(g<1)$ one [1]. For any $g<1$, the field $h$ drives FOQTs along the $h=0$ line. Relevant observables at the FOQT line are the energy difference $\Delta(h, L)$ of the lowest levels and the magnetization $m=L^{-1}\left\langle\sum_{i} \sigma_{i}^{(3)}\right\rangle$. In the following, we are interested in the behavior of the ground-state fidelity (2) arising from changes of the longitudinal field $h \equiv \lambda$, keeping $g$ fixed. The fidelity susceptibility is obtained by expanding $F$ to second order in powers of $\delta h$.

\section{A. Finite-size scaling at the continuous transition}

At the CQT, located at $g=1$ and $h=0$, the system is expected to develop the asymptotic FSS behavior in Eq. (8). Let us analyze two situations in which the control parameter is assumed to be either $h$ or $g$ and it is tuned through the CQT point.

We first consider the case in which the longitudinal field $h$ is varied across the value $h=0$, while the transverse field strength is kept fixed at $g=1$. The exponent $y_{h}$ entering the corresponding scaling variable $\kappa=h L^{y_{h}}$ [see Eq. (5)] is provided by the RG dimension of the longitudinal magnetic field $h$, i.e.,

$$
y_{h}=(d+z+2-\eta) / 2 .
$$

For the quantum Ising ring in Eq. (18), we have $d=1, z=1$, and $\eta=1 / 4$, thus $y_{h}=15 / 8$. Details on the derivation of the Ising critical exponents and of the RG dimension $y_{h}$ are provided, e.g., in Ref. [47]. Correspondingly, inserting such a value in Eq. (11) with $\lambda=h$, we find that, in the large- $L$ limit, the fidelity susceptibility diverges as

$$
\chi_{F}(h, L) \sim L^{15 / 4} \mathcal{F}_{2}(\kappa), \quad \kappa=h L^{15 / 8} .
$$

On the other hand, in the usual setting considered in the literature, the transverse field $g$ is varied across the value $g=1$ and the longitudinal field is kept fixed at $h=0$ [11,14,15,17-19]. In such case, an analogous FSS follows [17], where the scaling variable of Eq. (5) corresponding to the transverse field is $\kappa_{g}=(g-1) L^{y_{g}}$. For the quantum Ising chain, the RG dimension

$$
y_{g}=1 / v
$$

where $v=1$ (see again Ref. [47]). Therefore, Eq. (11) readily implies

$$
\chi_{F}(g, L) \sim L^{2} \mathcal{F}_{2}^{(g)}\left(\kappa_{g}\right), \quad \kappa_{g}=(g-1) L .
$$

\section{B. Finite-size scaling at the first-order transition}

The FOQTs, occurring at $g<1$ along the line $h=0$, can be related to the level crossing of the two lowest magnetized states $|+\rangle$ and $|-\rangle$ for $h=0$ such that $\left\langle \pm\left|\sigma_{i}^{(3)}\right| \pm\right\rangle= \pm m_{0}$, with $m_{0}=\left(1-g^{2}\right)^{1 / 8}$ [63]. Contrary to CQTs, the distinctive feature of FOQTs is a remarkable qualitative dependence of their features on the boundary conditions. As we will see below in a variety of different situations in the context of the Ising model, this also emerges in the FSS of the fidelity susceptibility, exhibiting completely different scalings, according to the size dependence of the energy difference of the lowest energy levels.

\section{Periodic or open boundary conditions}

In a finite system of size $L$ with periodic boundary conditions (PBCs) or open boundary conditions (OBCs), the lowest states are superpositions of $|+\rangle$ and $|-\rangle$, due to tunneling effects. Their energy difference $\Delta_{0}(L) \sim g^{L}$ vanishes exponentially with $L$. More precisely [64],

$$
\begin{gathered}
\Delta_{0}(L)=2\left(1-g^{2}\right) g^{L}\left[1+O\left(g^{2 L}\right)\right] \text { for OBC, } \\
\Delta_{0}(L) \approx 2 \sqrt{\left(1-g^{2}\right) / \pi L} g^{L} \text { for PBC. }
\end{gathered}
$$

Conversely, the difference $\Delta_{0, i} \equiv E_{i}-E_{0}$ for higher excited states $(i>1)$ remains finite for $L \rightarrow \infty$. The interplay of the size $L$ and the field $h$ gives rise to the FSS of the low-energy properties [48]. Its scaling variable is obtained from Eq. (13), i.e.,

$$
\kappa=\frac{2 m_{0} h L}{\Delta_{0}(L)},
$$

using the fact that $E_{h}=2 m_{0} h L$ is the energy variation associated with $h$. The FSS limit corresponds to $L \rightarrow \infty$ and $h \rightarrow 0$, keeping $\kappa$ fixed. Correspondingly, the energy difference of the lowest states and the magnetization behave as [48] $\Delta(h, L) \approx \Delta_{0}(L) \mathcal{D}(\kappa)$ and $m(L, h) \approx m_{0} \mathcal{M}(\kappa)$, where $\mathcal{D}(\kappa)$ and $\mathcal{M}(\kappa)$ are scaling functions independent of $g$. 


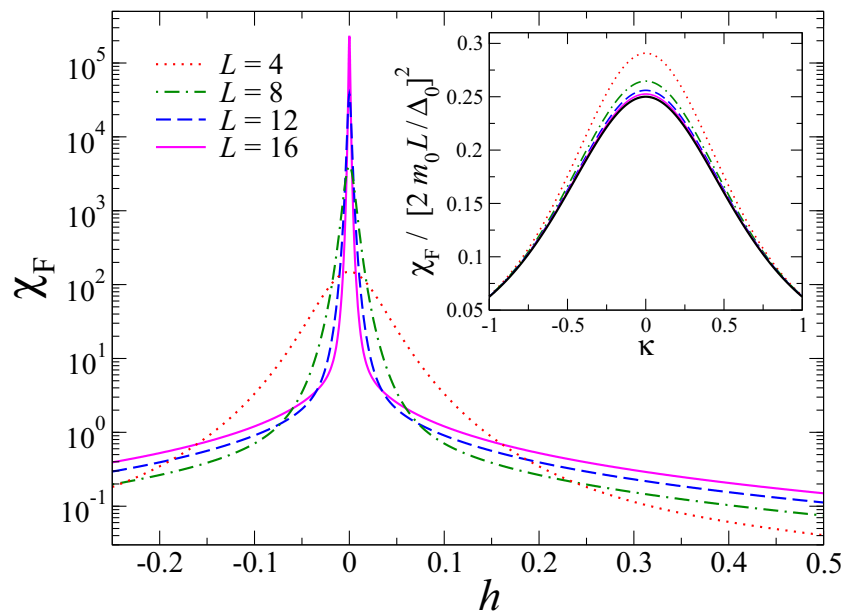

FIG. 1. Fidelity susceptibility $\chi_{F}(h, L)$ for the Ising model (18) with $g=0.9$ and PBC, associated with changes of the longitudinal parameter $h$, for several values of $L$, up to $L=16$. The inset displays curves for $\chi_{F} /\left[2 m_{0} L / \Delta_{0}(L)\right]^{2}$, as a function of $\kappa=2 m_{0} h L / \Delta_{0}(L)$ [see Eq. (26)], converging to the scaling function $\mathcal{F}_{2}^{(2 l)}(\kappa)$ (thick black line) [cf. Eq. (29)]. Analogous results are obtained for other value of $g<1$.

The FSS of the fidelity and its susceptibility is given by Eqs. (8) and (10). We obtain

$$
\chi_{F}(h, L) \approx\left[\frac{2 m_{0} L}{\Delta_{0}(L)}\right]^{2} \mathcal{F}_{2}(\kappa),
$$

implying that it exponentially diverges with $L$. This is confirmed by the numerical results ${ }^{2}$ of Fig. 1, where the curves of $\chi_{F}$ for PBCs display sharp, and exponentially increasing, peaks around $h=0$, while $\chi_{F}=O(L)$ for larger $|h|$.

Since the low-energy spectrum for PBCs and OBCs across the FOQT is characterized by the level crossing of the two lowest states, while the energy differences with the other ones remain $O(1)$, the asymptotic FSS can be exactly obtained by performing a two-level truncation of the spectrum [48-50], keeping only the lowest energy levels $| \pm\rangle$. Details are provided in the Appendix, where an extension to finite temperature is also presented, thus confirming Eq. (17). The net result is that, using the corresponding two-level effective Hamiltonian, we get

$$
\mathcal{F}^{(2 l)}(\kappa, \delta \kappa)=\cos (\delta \alpha / 2),
$$

where we defined

$$
\delta \alpha=\arctan \left[\frac{1}{\kappa+\delta \kappa}\right]-\arctan \frac{1}{\kappa},
$$

with $\arctan [x] \in(0, \pi)$. Moreover,

$$
\mathcal{F}_{2}^{(2 l)}(\kappa)=\frac{1}{4\left(1+\kappa^{2}\right)^{2}} .
$$

\footnotetext{
${ }^{2}$ Numerical results have been obtained by means of exact diagonalization (for $L \leqslant 12$ ) or through a Lanczos algorithm (for $L \geqslant 14$ ). The susceptibility has been extracted by quadratically fitting the fidelity as a function of $\delta h$, for $\delta h \lesssim 10^{-6}$.
}

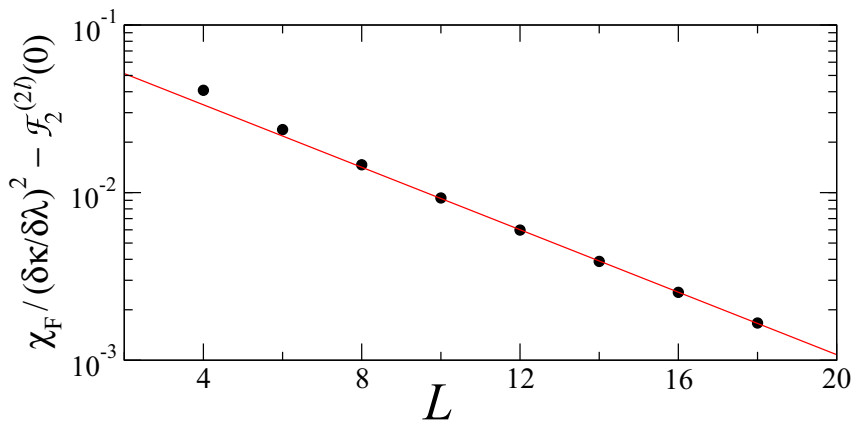

FIG. 2. Convergence of the finite-size fidelity susceptibility to the asymptotic scaling function $\mathcal{F}_{2}(\kappa)$. Data are for PBC, with $g=0.9$ and $\kappa=0$ (see Fig. 1). We plot the rescaled susceptibility $\chi_{F} /\left[2 m_{0} L / \Delta_{0}(L)\right]^{2}$ as a function of $L$, subtracting the asymptotic value given by $\mathcal{F}_{2}^{(2 l)}(0)=1 / 4$. The red line is an exponential fit of data for $5 \leqslant L \leqslant 18$.

The inset of Fig. 1 evidences the convergence of $\chi_{F} /\left[2 m_{0} L / \Delta_{0}(L)\right]^{2}$ to the scaling function $\mathcal{F}_{2}^{(2 l)}(\kappa)$, as a function of the scaling variable $\kappa$ in Eq. (25), which clearly turns out to be exponential, as shown by Fig. 2 .

\section{Antiperiodic boundary conditions}

As already mentioned, the FOQT scenario based on the avoided crossing of two levels, holding for PBCs and OBCs, is not always realized. Indeed, a quite different behavior emerges when considering antiperiodic boundary conditions (ABCs). This is essentially related to the fact that the corresponding low-energy states are one-kink (a nearest-neighbor pair of antiparallel spins) states, behaving as one-particle states with $O\left(L^{-1}\right)$ momenta. Thus, the energy difference of the lowest levels displays a power-law behavior [64]

$$
\Delta_{0}(L)=[g /(1-g)] \pi^{2} L^{-2}+O\left(L^{-4}\right) .
$$

Then, following Eq. (13), we can define the scaling variable

$$
\kappa=h L^{3} \text {. }
$$

Indeed, since the energy associated with the longitudinal field $h$ scales as $E_{h}(h, L) \sim h L$ and the gap $\Delta_{0}(L) \sim L^{-2}$, it is immediate to see that the ratio (13) obeys the same dependence on $h$ and $L$ as in Eq. (31).

The general ansatz (14) predicts a power-law behavior for the fidelity susceptibility,

$$
\chi_{F}(h, L) \approx L^{6} \mathcal{F}_{2}^{(a)}(\kappa),
$$

since $\partial \kappa / \partial h=L^{3}$. Again, this FSS is nicely supported by the numerical data ${ }^{2}$ of Fig. 3. With increasing $L$, the curves for the ratio $\chi_{F} / L^{6}$ appear to approach a scaling function $\mathcal{F}_{2}^{(a)}(\kappa)$. Finite-size corrections appear to be a power law, of $O\left(L^{-2}\right)$, as is visible from Fig. 4.

It is important to emphasize that, unlike the cases of PBCs and OBCs, for $\mathrm{ABCs}$ the scaling functions cannot be obtained by a two-level approximation, because the low-energy spectrum at the transition point presents a tower of excited stated with $\Delta_{0, i}=O\left(L^{-2}\right)$. We also note that, for $|h|>0, \chi_{F}$ 


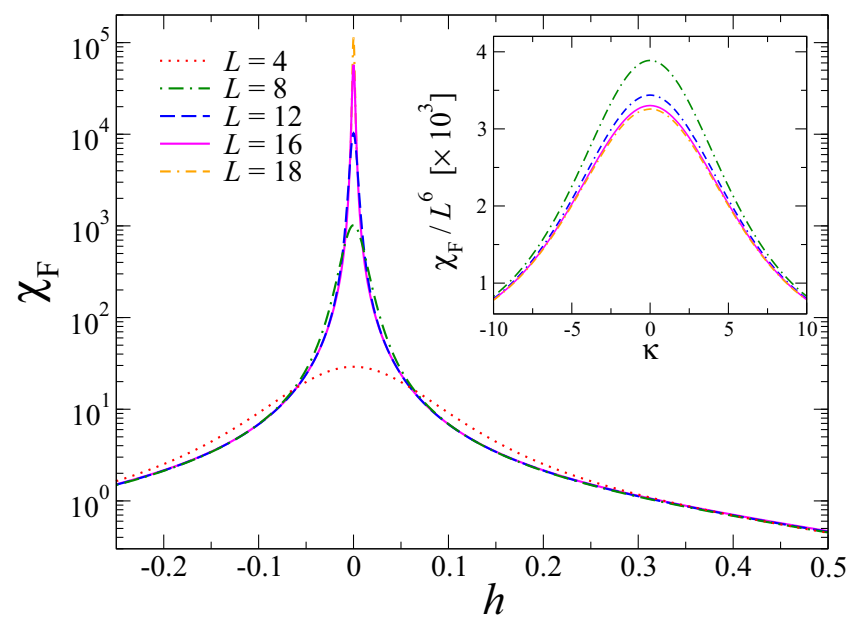

FIG. 3. Same as in Fig. 1, but for $g=0.5$ and ABCs. The inset shows the rescaled fidelity susceptibility according to Eq. (32), for $\kappa=h L^{3}$. The curves for $\chi_{F} / L^{6}$ clearly approach a scaling function of $\kappa$.

appears to converge to a finite value with increasing $L$, unlike for PBCs (compare the tails of Fig. 3 with those of Fig. 2).

\section{Equal and fixed boundary conditions}

Let us finally consider equal fixed boundary conditions (EFBCs) favoring one of the two magnetized phases. This is obtained by adding equal fixed spin states $|\downarrow\rangle$ at the ends $x=0$ and $x=L+1$ of the chain (18). In such a case, the interplay between the size $L$ and the bulk field $h$ gives rise to a more complex finite-size behavior with respect to that of neutral boundary conditions, such as PBCs and ABCs [53].

When $h=0$, the system is in the negatively magnetized phase and $\Delta_{0}(L)=4(1-g)+O\left(L^{-2}\right)$. For sufficiently small $h$, the observables depend smoothly on it. Then the system undergoes a sharp transition to the other phase at $h \approx h_{t r}(L)>0$, which tends to zero with increasing $L$, asymptotically as $h_{t r}(L) \approx \eta(g) / L$, where $\eta(g)$ is a $g$ dependent constant [53]. This sharp transition corresponds to the minimum $\Delta_{m}(L)$ of the energy difference $\Delta(h, L)$ of the lowest levels, which vanishes exponentially with increasing $L$,

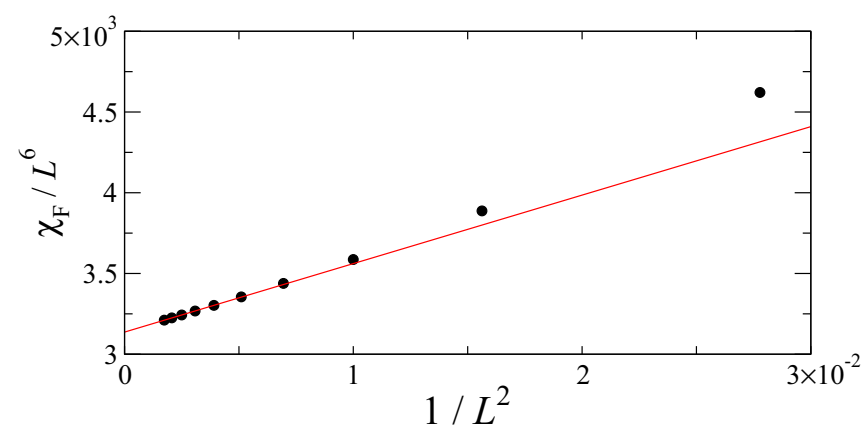

FIG. 4. Same as in Fig. 2 but for ABCs, with $g=0.5$ and $\kappa=0$ (see Fig. 3). Here we plot the rescaled susceptibility $\chi_{F} / L^{6}$ as a function of $L^{-2}$. The red line is a power-law fit of data for $14 \leqslant L \leqslant 24$.

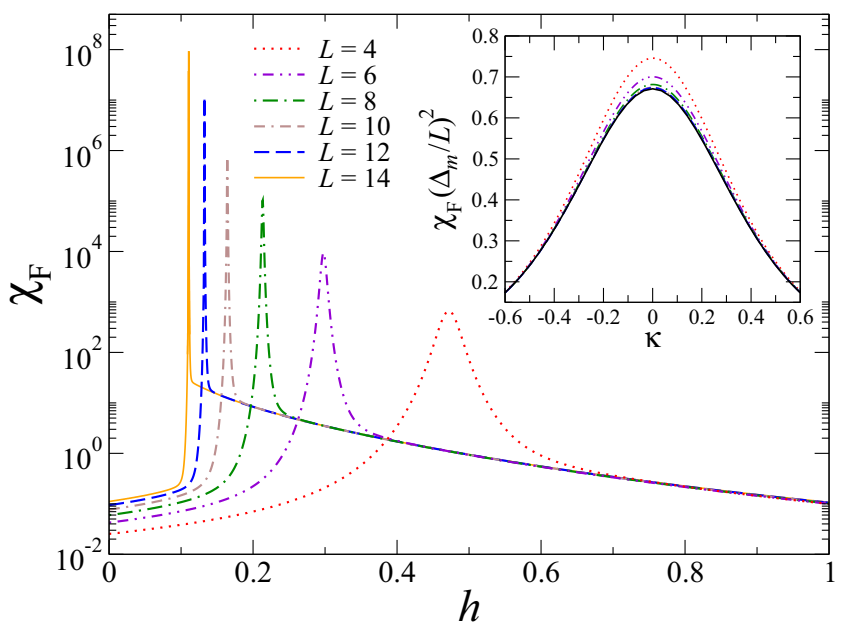

FIG. 5. Same as in Fig. 1 but for $g=0.5$ and EFBCs. The inset shows the FSS of $\chi_{F}$ around $h=h_{t r}(L)$, with $\kappa=\left[h-h_{t r}(L)\right] L / \Delta_{m}(L)$. With increasing $L$, the curves of $\left(\Delta_{m} / L\right)^{2} \chi_{F}(h, L)$ rapidly (exponentially) approach the two-level scaling function [cf. Eq. (34)], with $a \approx 0.67$ and $b \approx 1.64$.

as $\Delta_{m}(L) \sim e^{-b(g) L}$. Around $h_{t r}$, the suitable scaling variable turns out to be

$$
\kappa=\frac{\left[h-h_{t r}(L)\right] L}{\Delta_{m}(L)},
$$

analogously to that of PBCs and OBCs, apart from the $1 / L$ shift of the transition point. The corresponding scaling behaviors, $\Delta(h, L) \approx \Delta_{m}(L) \mathcal{D}(\kappa)$ and $m(h, L) \approx m_{0} \mathcal{M}(\kappa)$, turn out to be those emerging from an avoided two-level crossing, similarly to the case of PBCs.

Figure 5 shows the $h$ dependence of the fidelity susceptibility $\chi_{F}(h, L)$ for several values of $L .^{2}$ Its behavior reflects that of other observables. In particular, it is smooth around $h=0$, since we checked that the ratio $\chi_{F}(h, L) / \chi_{F}(h=0, L)$ rapidly approaches a function of $h$ only, with $\chi_{F}(0, L)=O(L)$ (not shown). Then, with increasing $h$, the curves show a sharp peak around $h_{t r}(L)$ whose maximum rapidly increases with $L$ and becomes narrower and narrower. For even larger $h, \chi_{F}(h, L)$ tends to rapidly become independent of $L$; this is related to the fact that the ground state is essentially given by spatially separated kink and antikink structures whose position depends smoothly on $h$ [53]. The scaling behavior around $h_{t r}(L)$ can be inferred from the general ansatz (10),

$$
\frac{\Delta_{m}^{2}}{L^{2}} \chi_{F}(h, L) \approx a \mathcal{F}_{2}^{(2 l)}(b \kappa),
$$

where the scaling variable $\kappa$ is that given in Eq. (33), $\mathcal{F}_{2}^{(2 l)}(x)$ is the two-level scaling function (29), and $a$ and $b$ are appropriate normalizations. This is confirmed by numerical data in the inset of Fig. 5.

Finally, we mention that the case of fixed, but opposite, boundary conditions (OFBCs), i.e., $|\downarrow\rangle$ and $|\uparrow\rangle$ at the ends of the chain, is supposed to be similar to that with ABCs [51,52], because the low-energy states are again one-kink states. Thus, $\Delta_{0}(L) \sim L^{-2}$ as well, and a power-law behavior such as (32) is expected. 


\section{CONCLUSION}

We have shown that the ground-state fidelity and the corresponding susceptibility develop FSS behaviors at both CQTs and FOQTs, arising from the interplay between the driving parameter and the system size. At CQTs the fidelity susceptibility generally shows power laws: $\chi_{F} \sim L^{2 y_{\lambda}}$ at the transition point, where $y_{\lambda}$ is the universal exponent associated with the critical properties of the corresponding Hamiltonian perturbation. At CQTs boundary conditions only affect the scaling functions of observables. This sharply contrasts with FOQTs, whose distinctive feature is a remarkable qualitative dependence on the boundary conditions; indeed, the fidelity susceptibility may show exponential or power-law FSS, essentially related to the size dependence of the energy difference of the lowest levels. In particular, exponential behaviors develop for boundary conditions such as PBCs or EFBCs, for which $\chi_{F} \sim e^{c L^{d}}$ at the transition point. Conversely, power-law behaviors similar to those occurring at CQTs turn out to develop for $\mathrm{ABCs}$ or for OFBCs. Our findings have been confirmed by analytical and numerical results for the one-dimensional quantum Ising model.

It is worth mentioning that the FSS treatment adopted here for the study of the ground-state fidelity in the quantum Ising ring shares important similarities with the approach previously employed to address other quantities in different kinds of QTs. First of all, the definition of the relevant scaling variable $\kappa$ through Eqs. (5) and (13) (for first-order and for continuous QTs, respectively) is closely related to the general arguments put forward in Refs. [2,47] for CQTs and in Ref. [48] for FOQTs. Moreover, the striking dependence of the FSS behavior at FOQTs (here evidenced for the fidelity) has been spotlighted in similar contexts as well, for lowlying energy gaps, local observables, and correlation functions [51-53], yielding consistent results.

All these connections are in support of the broad validity of our FSS theory: Indeed, we expect it to hold even in higher dimensions and for FOQTs of other models, where it would be tempting to have a direct numerical validation. Moreover, the possibility to generalize it to finite temperature makes it relevant also to quantum thermometry close to criticality, where estimation performances depend on the scaling behavior [9]. We also notice that the FSS frameworks have been extended to the off-equilibrium quantum dynamics, focusing on both time-dependent perturbations [49] and sudden quenches [50]. By defining scaling variables that are consistent with the procedure considered in this paper and including further ones associated with the time and the dynamic variables, dynamic FSS behaviors have been shown to emerge even in other contexts, as for the decoherence properties [65] and the statistics of the work [66].

As suggested from the present study, the FSS of the fidelity is amenable to a direct experimental verification by means of small-size quantum simulators (i.e., of the order of ten spins), which can thus serve as a probe of the nature of the transition itself. A possible strategy would be to measure the Loschmidt echo after a sudden quench $[67,68]$, a quantity strictly related to the fidelity susceptibility [69-72], which might shed light on the mutual interplay between QTs, entanglement, and decoherence [73-75].

\section{ACKNOWLEDGMENTS}

We thank R. Fazio and A. Pelissetto for fruitful discussions.

\section{APPENDIX: TWO-LEVEL REDUCTION OF THE SPECTRUM ACROSS FOQT LINE}

As stated in Sec. III B 1, in the thermodynamic limit, the low-energy spectrum for PBCs and for OBCs across the FOQT is characterized by the level crossing of the two lowest states, while the energy differences with the other ones remain finite. The asymptotic FSS behavior for the fidelity and for its susceptibility can be thus exactly obtained by performing a two-level truncation of the spectrum, following Refs. [47,49,50], keeping only the lowest energy levels. For the sake of completeness, here we sketch this derivation.

The effective Hamiltonian, written in the Hilbert space spanned by the two lowest magnetized states $|+\rangle$ and $|-\rangle$ for $h=0$, i.e., such that $\left\langle \pm\left|\sigma_{i}^{(3)}\right| \pm\right\rangle= \pm m_{0} \quad$ [with $\left.m_{0}=\left(1-g^{2}\right)^{1 / 8}\right]$, reads

$$
H_{2}(h)=-\beta \sigma^{(3)}+\delta \sigma^{(1)} .
$$

The parameters $\beta$ and $\delta$ correspond to $\beta=m_{0} h L$ and $\delta=\Delta_{0} / 2$ such that $\kappa(h)=\beta / \delta$. The eigenstates are

$$
\begin{aligned}
& |0\rangle=\sin (\alpha / 2)|-\rangle-\cos (\alpha / 2)|+\rangle, \\
& |1\rangle=\cos (\alpha / 2)|-\rangle+\sin (\alpha / 2)|+\rangle,
\end{aligned}
$$

where $\tan \alpha=\kappa^{-1}$ with $\alpha \in(0, \pi)$ and $E_{1}-E_{0}=\Delta_{0} \sqrt{1+\kappa^{2}}$.

Straightforward calculations confirm the FSS behavior in Eq. (8) of the zero-temperature fidelity:
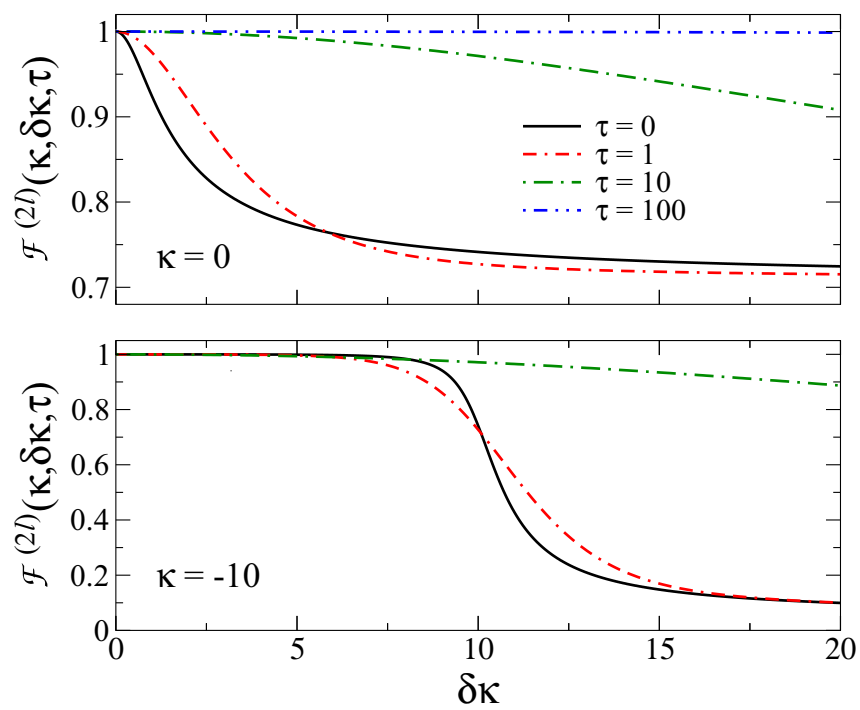

FIG. 6. Scaling function of the fidelity susceptibility for two different values of $\kappa$, at finite temperature $\tau$, as obtained in a twolevel truncation scheme. The solid black curves correspond to the zero-temperature case, for which the analytic curve of Eq. (A4) holds. 
$F(\lambda, \delta \lambda, L) \approx \mathcal{F}(\kappa, \delta \kappa)$. Indeed, we obtain

$$
F(h, \delta h, L) \approx \mathcal{F}^{(2 l)}(\kappa, \delta \kappa)=\cos (\delta \alpha / 2),
$$

where $\tan (\alpha+\delta \alpha)=(\kappa+\delta \kappa)^{-1}$. The corresponding scaling function in Eq. (29) of the fidelity susceptibility is thus easily obtained.

As discussed in Sec. II, the definition of fidelity can be extended to finite temperature as well, through Eq. (16). The computation based on the two-level truncation confirms the
FSS behavior put forward in Eq. (17). In Fig. 6 we plot the scaling function $\mathcal{F}^{(2 l)}(\kappa, \delta \kappa, \tau)$ for different values of $\kappa$ and $\tau$. Note that, for $\kappa=0$, the zero-temperature fidelity at large $\delta \kappa$ approaches the asymptotic value $|\langle+\mid 0\rangle|=2^{-1 / 2} \approx 0.707$. On the other hand, for $\kappa \rightarrow-\infty$, it approaches zero, since it corresponds to abruptly sweeping from one side of the transition to the other. The effect of the temperature is to progressively smoothen the behavior of the various curves with $\delta \kappa$
[1] S. Sachdev, Quantum Phase Transitions (Cambridge University, Cambridge, 1999).

[2] S. L. Sondhi, S. M. Girvin, J. P. Carini, and D. Shahar, Continuous quantum phase transitions, Rev. Mod. Phys. 69, 315 (1997).

[3] L. Amico, R. Fazio, A. Osterloh, and V. Vedral, Entanglement in many-body systems, Rev. Mod. Phys. 80, 517 (2008).

[4] S.-J. Gu, Fidelity approach to quantum phase transitions, Int. J. Mod. Phys. B 24, 4371 (2010).

[5] D. Braun, G. Adesso, F. Benatti, R. Floreanini, U. Marzolino, M. W. Mitchell, and S. Pirandola, Quantum-enhanced measurements without entanglement, Rev. Mod. Phys. 90, 035006 (2018).

[6] P. W. Anderson, Infrared Catastrophe in Fermi Gases with Local Scattering Potentials, Phys. Rev. Lett. 18, 1049 (1967).

[7] S. L. Braunstein and C. M. Caves, Statistical Distance and the Geometry of Quantum States, Phys. Rev. Lett. 72, 3439 (1994).

[8] M. G. A. Paris, Quantum estimation for quantum technology, Int. J. Quantum Inf. 7, 125 (2009).

[9] P. Zanardi, M. G. A. Paris, and L. Campos Venuti, Quantum criticality as a resource for quantum estimation, Phys. Rev. A 78, 042105 (2008).

[10] C. Invernizzi, M. Korbman, L. Campos Venuti, and M. G. A. Paris, Optimal quantum estimation in spin systems at criticality, Phys. Rev. A 78, 042106 (2008).

[11] P. Zanardi and N. Paunković, Ground state overlap and quantum phase transitions, Phys. Rev. E 74, 031123 (2006).

[12] W.-L. You, Y.-W. Li, and S.-J. Gu, Fidelity, dynamic structure factor, and susceptibility in critical phenomena, Phys. Rev. E 76, 022101 (2007).

[13] L. Campos Venuti and P. Zanardi, Quantum Critical Scaling of the Geometric Tensors, Phys. Rev. Lett. 99, 095701 (2007).

[14] M. Cozzini, P. Giorda, and P. Zanardi, Quantum phase transitions and quantum fidelity in free fermion graphs, Phys. Rev. B 75, 014439 (2007).

[15] M. M. Rams and B. Damski, Quantum Fidelity in the Thermodynamic Limit, Phys. Rev. Lett. 106, 055701 (2011); Scaling of ground state fidelity in the thermodynamic limit: $X Y$ model and beyond, Phys. Rev. A 84, 032324 (2011).

[16] V. Mukherjee, A. Polkovnikov, and A. Dutta, Oscillating fidelity susceptibility near a quantum multicritical point, Phys. Rev. B 83, 075118 (2011).

[17] B. Damski, Fidelity susceptibility of the quantum Ising model in the transverse field: The exact solution, Phys. Rev. E 87, 052131 (2013).

[18] Q. Luo, J. Zhao, and X. Wang, Fidelity susceptibility of the anisotropic $X Y$ model: The exact solution, Phys. Rev. E 98, 022106 (2018).
[19] S. Chen, L. Wang, Y. Hao, and Y. Wang, Intrinsic relation between ground-state fidelity and the characterization of a quantum phase transition, Phys. Rev. A 77, 032111 (2008).

[20] D. Schwandt, F. Alet, and S. Capponi, Quantum Monte Carlo Simulations of Fidelity at Magnetic Quantum Phase Transitions, Phys. Rev. Lett. 103, 170501 (2009).

[21] B. Li, S.-H. Li, and H.-Q. Zhou, Quantum phase transitions in a two-dimensional quantum $X Y X$ model: Ground-state fidelity and entanglement, Phys. Rev. E 79, 060101(R) (2009).

[22] A. F. Albuquerque, F. Alet, C. Sire, and S. Capponi, Quantum Critical Scaling of Fidelity Susceptibility, Phys. Rev. B 81, 064418 (2010).

[23] J. Sirker, Finite-Temperature Fidelity Susceptibility for OneDimensional Quantum Systems, Phys. Rev. Lett. 105, 117203 (2010).

[24] Y. Nishiyama, Criticalities of the transverse- and longitudinalfield fidelity susceptibilities for the $d=2$ quantum Ising model, Phys. Rev. E 88, 012129 (2013).

[25] G. Sun, A. K. Kolezhuk, and T. Vekua, Fidelity at BerezinskiiKosterlitz-Thouless quantum phase transitions, Phys. Rev. B 91, 014418 (2015).

[26] P. Buonsante and A. Vezzani, Ground-State Fidelity and Bipartite Entanglement in the Bose-Hubbard Model, Phys. Rev. Lett. 98, 110601 (2007).

[27] S. R. Manmana, K. R. A. Hazzard, G. Chen, A. E. Feiguin, and A. M. Rey, $\mathrm{SU}(N)$ magnetism in chains of ultracold alkalineearth-metal atoms: Mott transitions and quantum correlations, Phys. Rev. A 84, 043601 (2011).

[28] J. Carrasquilla, S. R. Manmana, and M. Rigol, Scaling of the gap, fidelity susceptibility, and Bloch oscillations across the superfluid to Mott insulator transition in the one-dimensional Bose-Hubbard model, Phys. Rev. A 87, 043606 (2013).

[29] L. Wang, Y.-H. Liu, J. Imriška, P. N. Ma, and M. Troyer, Fidelity Susceptibility Made Simple: A Unified Quantum Monte Carlo Approach, Phys. Rev. X 5, 031007 (2015).

[30] L. Huang, Y. Wang, L. Wang, and P. Werner, Detecting phase transitions and crossovers in Hubbard models using the fidelity susceptibility, Phys. Rev. B 94, 235110 (2016).

[31] S. Kettemann, Exponential Orthogonality Catastrophe at the Anderson Metal-Insulator Transition, Phys. Rev. Lett. 117, 146602 (2016).

[32] S. Santhosh Kumar and S. Shankaranarayanan, Evidence of quantum phase transition in real-space vacuum entanglement of higher derivative scalar quantum field theories, Sci. Rep. 7, 15774 (2017).

[33] S. Yang, S.-J. Gu, C.-P. Sun, and H.-Q. Lin, Fidelity susceptibility and long-range correlation in the Kitaev honeycomb model, Phys. Rev. A 78, 012304 (2008). 
[34] T. P. Oliveira and P. D. Sacramento, Entanglement modes and topological phase transitions in superconductors, Phys. Rev. B 89, 094512 (2014).

[35] E. J. König, A. Levchenko, and N. Sedlmayr, Universal fidelity near quantum and topological phase transitions in finite onedimensional systems, Phys. Rev. B 93, 235160 (2016).

[36] L. Banchi, P. Giorda, and P. Zanardi, Quantum informationgeometry of dissipative quantum phase transitions, Phys. Rev. E 89, 022102 (2014).

[37] U. Marzolino and T. Prosen, Fisher information approach to nonequilibrium phase transitions in a quantum XXZ spin chain with boundary noise, Phys. Rev. B 96, 104402 (2017).

[38] V. Piazza, V. Pellegrini, F. Beltram, W. Wegscheider, T. Jungwirth, and A. H. MacDonald, First-order phase transitions in a quantum Hall ferromagnet, Nature (London) 402, 638 (1999).

[39] T. Vojta, D. Belitz, T. R. Kirkpatrick, and R. Narayanan, Quantum critical behavior of itinerant ferromagnets, Ann. Phys. (Leipzig) 8, 593 (1999).

[40] M. Uhlarz, C. Pfleiderer, and S. M. Hayden, Quantum Phase Transitions in the Itinerant Ferromagnet $\mathrm{ZrZn}_{2}$, Phys. Rev. Lett. 93, 256404 (2004).

[41] C. Pfleiderer, Why first order quantum phase transitions are interesting, J. Phys.: Condens. Matter 17, S987 (2005).

[42] W. Knafo, S. Raymond, P. Lejay, and J. Flouquet, Antiferromagnetic criticality at a heavy-fermion quantum phase transition, Nat. Phys. 5, 753 (2009).

[43] T. Jörg, F. Krzakala, J. Kurchan, and A. C. Maggs, Simple Glass Models and their Quantum Annealing, Phys. Rev. Lett. 101, 147204 (2008).

[44] T. Jörg, F. Krzakala, G. Semerjian, and F. Zamponi, First-Order Transitions and the Performance of Quantum Algorithms in Random Optimization Problems, Phys. Rev. Lett. 104, 207206 (2010).

[45] A. P. Young, S. Knysh, and V. N. Smelyanskiy, First Order Phase Transition in the Quantum Adiabatic Algorithm, Phys. Rev. Lett. 104, 020502 (2010).

[46] Y. Seki and H. Nishimori, Quantum annealing with antiferromagnetic fluctuations, Phys. Rev. E 85, 051112 (2012).

[47] M. Campostrini, A. Pelissetto, and E. Vicari, Finite-size scaling at quantum transitions, Phys. Rev. B 89, 094516 (2014).

[48] M. Campostrini, J. Nespolo, A. Pelissetto, and E. Vicari, FiniteSize Scaling at First-Order Quantum Transitions, Phys. Rev. Lett. 113, 070402 (2014); Finite-size scaling at first-order quantum transitions of quantum Potts chains, Phys. Rev. E 91, 052103 (2015).

[49] A. Pelissetto, D. Rossini, and E. Vicari, Off-equilibrium dynamics driven by localized time-dependent perturbations at quantum phase transitions, Phys. Rev. B 97, 094414 (2018).

[50] A. Pelissetto, D. Rossini, and E. Vicari, Dynamic finite-size scaling after a quench at quantum transitions, Phys. Rev. E 97, 052148 (2018).

[51] M. Campostrini, A. Pelissetto, and E. Vicari, Quantum transitions driven by one-bond defects in quantum Ising rings, Phys. Rev. E 91, 042123 (2015).

[52] M. Campostrini, A. Pelissetto, and E. Vicari, Quantum Ising chains with boundary fields, J. Stat. Mech. (2015) P11015.

[53] A. Pelissetto, D. Rossini, and E. Vicari, Finite-size scaling at first-order quantum transitions when boundary conditions favor one of the two phases, Phys. Rev. E 98, 032124 (2018).
[54] P. Calabrese, J. Cardy, and B. Doyon, Entanglement entropy in extended systems, J. Phys. A: Math. Theor. 42, 500301 (2009).

[55] G. De Chiara and A. Sanpera, Genuine quantum correlations in quantum many-body systems: A review of recent progress, Rep. Prog. Phys. 81, 074002 (2018).

[56] M. N. Barber, in Phase Transitions and Critical Phenomena, edited by C. Domb and J. L. Lebowitz (Academic, London 1983), Vol. 8, p. 145.

[57] Finite Size Scaling and Numerical Simulations of Statistical Systems, edited by V. Privman (World Scientific, Singapore, 1990).

[58] A. Pelissetto and E. Vicari, Critical phenomena and renormalization-group theory, Phys. Rep. 368, 549 (2002).

[59] F. M. Gasparini, M. O. Kimball, K. P. Mooney, and M. DiazAvila, Finite-size scaling of ${ }^{4} \mathrm{He}$ at the superfluid transition, Rev. Mod. Phys. 80, 1009 (2008).

[60] I. Bloch, J. Dalibard, and W. Zwerger, Many-body physics with ultracold gases, Rev. Mod. Phys. 80, 885 (2008).

[61] M. E. Fisher and M. N. Barber, Scaling Theory for FiniteSize Effects in the Critical Region, Phys. Rev. Lett. 28, 1516 (1972).

[62] A. Uhlmann, The "transition probability" in the state space of a *-algebra, Rep. Math. Phys. 9, 273 (1976).

[63] P. Pfeuty, The one-dimensional Ising model with a transverse field, Ann. Phys. (NY) 57, 79 (1970).

[64] G. G. Cabrera and R. Jullien, Role of boundary conditions in the finite-size Ising model, Phys. Rev. B 35, 7062 (1987).

[65] E. Vicari, Decoherence dynamics of qubits coupled to systems at quantum transitions, Phys. Rev. A 98, 052127 (2018).

[66] D. Nigro, D. Rossini, and E. Vicari, Scaling properties of work fluctuations after quenches at quantum transitions, arXiv:1810.04614.

[67] J. Zhang, X. Peng, N. Rajendran, and D. Suter, Detection of Quantum Critical Points by a Probe Qubit, Phys. Rev. Lett. 100, 100501 (2008).

[68] J. Zhang, F. M. Cucchietti, C. M. Chandrashekar, M. Laforest, C. A. Ryan, M. Ditty, A. Hubbard, J. K. Gamble, and R. Laflamme, Direct observation of quantum criticality in Ising spin chains, Phys. Rev. A 79, 012305 (2009).

[69] T. Gorin, T. Prosen, T. H. Seligman, and M. Žnidarič, Dynamics of Loschmidt echoes and fidelity decay, Phys. Rep. 435, 33 (2006).

[70] P. Jacquod and C. Petitjean, Decoherence, entanglement and irreversibility in quantum dynamical systems with few degrees of freedom, Adv. Phys. 58, 67 (2009).

[71] C. De Grandi, V. Gritsev, and A. Polkovnikov, Quench dynamics near a quantum critical point, Phys. Rev. B 81, 012303 (2010).

[72] M. Serbyn and D. A. Abanin, Loschmidt echo in many-body localized phases, Phys. Rev. B 96, 014202 (2017).

[73] H. T. Quan, Z. Song, X. F. Liu, P. Zanardi, and C. P. Sun, Decay of Loschmidt Echo Enhanced by Quantum Criticality, Phys. Rev. Lett. 96, 140604 (2006).

[74] D. Rossini, T. Calarco, V. Giovannetti, S. Montangero, and R. Fazio, Decoherence induced by interacting quantum spin baths, Phys. Rev. A 75, 032333 (2007).

[75] P. Haikka, J. Goold, S. McEndoo, F. Plastina, and S. Maniscalco, Non-Markovianity, Loschmidt echo, and criticality: A unified picture, Phys. Rev. A 85, 060101(R) (2012). 